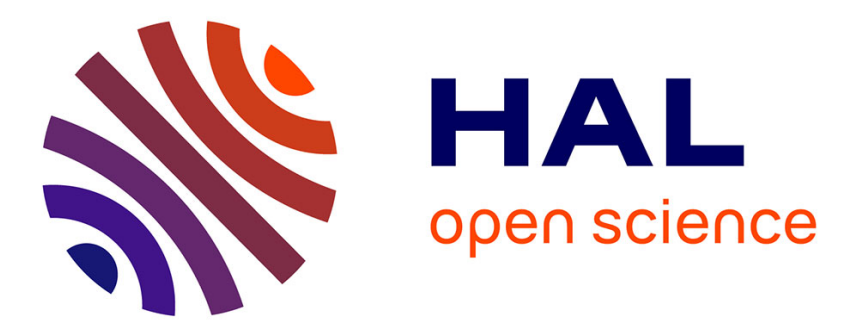

\title{
Microalgal lipid droplets: composition, diversity, biogenesis and functions
}

Hugh Goold, Fred Beisson, G. Peltier, Yonghua Li-Beisson

\section{To cite this version:}

Hugh Goold, Fred Beisson, G. Peltier, Yonghua Li-Beisson. Microalgal lipid droplets: composition, diversity, biogenesis and functions. Plant Cell Reports, 2015, 34 (4), pp.545-555. 10.1007/s00299014-1711-7 . hal-03378474

\section{HAL Id: hal-03378474 https://hal.science/hal-03378474}

Submitted on 14 Oct 2021

HAL is a multi-disciplinary open access archive for the deposit and dissemination of scientific research documents, whether they are published or not. The documents may come from teaching and research institutions in France or abroad, or from public or private research centers.
L'archive ouverte pluridisciplinaire $\mathbf{H A L}$, est destinée au dépôt et à la diffusion de documents scientifiques de niveau recherche, publiés ou non, émanant des établissements d'enseignement et de recherche français ou étrangers, des laboratoires publics ou privés. 


\title{
Microalgal lipid droplets: composition, diversity, biogenesis and functions
}

\author{
Hugh Goold · Fred Beisson · Gilles Peltier • \\ Yonghua Li-Beisson
}

Received: 6 October 2014/Revised: 12 November 2014/ Accepted: 19 November 2014

(C) Springer-Verlag Berlin Heidelberg 2014

\begin{abstract}
Lipid droplet is the major site of neutral lipid storage in eukaryotic cells, and increasing evidence show its involvement in numerous cellular processes such as lipid homeostasis, signaling, trafficking and inter-organelle communications. Although the biogenesis, structure, and functions of lipid droplets have been well documented for seeds of vascular plants, mammalian adipose tissues, insects and yeasts, relative little is known about lipid droplets in microalgae. Over the past 5 years, the growing interest of microalgae as a platform for biofuel, green chemicals or value-added polyunsaturated fatty acid production has brought algal lipid droplets into spotlight. Studies conducted on the green microalga Chlamydomonas reinhardtii and other model microalgae such as Haematococcus and Nannochloropsis species have led to the identification of proteins associated with lipid droplets, which include putative structural proteins different from plant oleosins and animal perilipins, as well as candidate proteins for lipid biosynthesis, mobilization, trafficking and
\end{abstract}

Communicated by Neal Stewart.

H. Goold · F. Beisson - G. Peltier · Y. Li-Beisson CEA, IBEB, Lab Bioenerget Biotechnol Bacteries and Microalgues, Saint-Paul-lez-Durance 13108, France

H. Goold · F. Beisson - G. Peltier · Y. Li-Beisson

CNRS, UMR 7265 Biol Veget and Microbiol Environ,

Saint-Paul-lez-Durance 13108, France

H. Goold · F. Beisson · G. Peltier · Y. Li-Beisson ( $\square)$

Aix Marseille Université, BVME UMR7265,

Marseille 13284, France

e-mail: yonghua.li@cea.fr

H. Goold

Faculty of Agriculture and the Environment, University of

Sydney, Sydney, Australia homeostasis. Biochemical and microscopy studies have also started to shed light on the role of chloroplasts in the biogenesis of lipid droplets in Chlamydomonas.

Keywords Microalgal oil - Major lipid droplet protein · Oil bodies $\cdot$ Lipid homeostasis $\cdot$ Lipid turnover

\section{Introduction}

Due to increasing interests in the production of renewable energies from microalgae, microalgal oil metabolism is subjected to intensive research (Liu and Benning 2013; Merchant et al. 2012). All eukaryotic microalgae examined so far can produce oils as energy storage, either prior to a forthcoming dormancy period or subjected to unfavorable environmental conditions ( $\mathrm{Hu}$ et al. 2008). Since oils are carbon dense molecules and are non-polar, they are stored in hydrophobic subcellular structures called lipid droplets, also named as lipid bodies, oil bodies, oil globules, or oleosomes (Huang 1996; Murphy 2001). The choice of one name versus the other tends to be community-biased, and does not reflect any differences in their compositions. For example, the plant oil community tends to use more oil bodies, whereas the emerging algae lipid community adopted often lipid droplet. For simplicity reasons, only lipid droplet (LD) is used throughout this review.

LDs have been initially studied mostly in oil-rich tissues/organs, like adipose tissue, oilseeds, or nitrogen starved cells (Czabany et al. 2007; Goodman 2008; Huang 1996; Murphy 2001; Zweytick et al. 2000). With the development in new analytical and visualization techniques, it is now generally accepted that LDs are present in most eukaryotic cells and play a large array of cellular functions (Farese and Walther 2009). These studies led to 
the conclusion that rather than a mere energy storage depot, LDs are actually dynamic structures at the heart of lipid and energy metabolism (Farese and Walther 2009). Functions associated with LDs include but are not limited to lipid synthesis, degradation, vesicle trafficking, lipid secretion and lipid-based signaling. Only recently has research been carried out on LDs isolated from vegetative tissues of plants (Chapman et al. 2012; Horn et al. 2013; Shimada et al. 2014) or photosynthetic microorganisms. Here, we review the emerging literature on the composition, function and biogenesis of LDs in a variety of eukaryotic microalgae. Reference to other organisms is made only in a comparative context.

\section{Occurrence, formation and dynamics}

The occurrence and formation of LDs can be traced easily by microscopic examination or through flow cytometry, taking advantage of the lipophilic property of dyes such as Nile red or Bodipy (Cagnon et al. 2013; Greenspan et al. 1985; Mou et al. 2012; Xie et al. 2014; Yan et al. 2013; Zhang et al. 2014). Structure of LDs has been studied under Confocal fluorescent or Transmission Electron Microscopes (TEM) (Fig. 1) (Fan et al. 2011; Goodson et al. 2011; Wang et al. 2009). The size of LDs varies depending on species, growth stage and environmental conditions. It ranges from a dozen of nanometers to $30 \mu \mathrm{m}$. For example, cells of Chlamydomonas reinhardtii make LDs of around $1.5 \mu \mathrm{m}$ (Moellering and Benning 2010), whereas in Chlorella sp., the LDs have a diameter of $>3 \mu \mathrm{m}$ (Lin et al. 2012); in plant seeds and yeasts, a diameter of $0.5-2 \mu \mathrm{m}$ is usually reported, whereas in adipose tissue, LDs normally have a size of $2-10 \mu \mathrm{m}$; the largest LDs observed are in mesocarp tissues where they range from 10 to $20 \mu \mathrm{m}$. These size variations together with other features of lipid droplets isolated from diverse algal species are summarized in Table 1. In multicellular organisms, the size of LDs is postulated to be related to their specific biological functions and to the type of tissue. For example, in some animal cells, it is used as an insulation barrier to cold; and in oil seeds/algae cells, it is used as an energy source for germination and vegetative growth (Graham 2008). LDs present in adipose tissues tends to have large diameter, where in the latter case, smaller droplet allows higher ratio of surface area per unit of triacylglycerols (TAGs), thus facilitating access of enzymes (i.e., lipases) for mobilization of oils stored in LDs (Tzen et al. 1993).

In microalgae, one or two LDs per cell are usually present in nutrient replete conditions. Both the number and size of lipid droplets increase upon being exposed to a stress condition; and LDs can continue to increase in size as stress prolongs. This process is accompanied by degradation of membrane lipids as can be evidenced in the disorganization of thylakoid grana under TEM. Once culture conditions become favorable again, the accumulated LDs disappear as quickly as they are synthesized (usually completely remobilized after $48 \mathrm{~h}$ ) (Li et al. 2012b; Siaut et al. 2011). Simultaneous to this process is the re-syntheses of photosynthetic membranes and regain of vegetative growth. A phenomenon similar to what happens in plant oilseeds, where during germination, stored oils in seeds are mobilized to produce sugars important for germination and seedling establishment (Eastmond 2006; Graham 2008). This is, however, contrary to fruit mesocarp of olives, avocado and oil palm, where oils contained in LDs are the 'end product' of the lipid metabolism and are not mobilized by the host cells (Bourgis et al. 2011; Chapman et al. 2012). They are rather used by the host cell as 'baits' to attract potential predators, therefore ensuring efficient seed dispersal.

A large accumulation of lipid droplets has been found to be triggered by nutrient starvation (-N, - Fe, $-\mathrm{S},-\mathrm{P})$, high light, hypoxia, increased salinity, and also by chemical treatment (for example, Brefeldin A) (Boyle et al. 2012; Hemschemeier et al. 2013; Kato et al. 2013; Kim et al. 2013; Siaut et al. 2011; Urzica et al. 2013; Wang et al. 2009). Among various triggers, the best studied is the $\mathrm{N}$-starvation response. Transcriptomic and metabolic studies carried out in cells starved for nitrogen have shown that massive re-orientation of cellular metabolism toward carbon reserve formation (starch and oils) occurs at the onset of nitrogen starvation; this effect being accompanied by the down-regulation of genes involved in protein synthesis and photosynthesis (Boyle et al. 2012; Miller et al. 2010). Besides the up-regulation of genes encoding enzymes essential for conversion of acetate to fatty acyl chains, numerous lipases with increased transcription were also observed, which supported the notion that newly formed TAGs are partly coming from de novo synthesis and partly from membrane lipid remodeling (Fan et al. 2011; Moellering and Benning 2010; Siaut et al. 2011).

\section{Structure and composition}

The current structural model of a LD consists of a neutral lipid core (usually TAGs), which are surrounded by a monolayer of polar lipids (PLs) and are decorated by proteins. This model was originally proposed for maize grains (Tzen and Huang 1992), but it seems to hold true for LDs isolated from diverse kingdoms (Czabany et al. 2008; Yang et al. 2012; Zweytick et al. 2000). TAGs and PLs can take up to $90 \%$ of total weight, whereas proteins weigh between 1 and $5 \%$ (by weight). Depending on species, some minor components including free fatty acids, carotenes, etc, are also present. No sugars have so far been reported to be associated with LDs. The ratio between 




Fig. 1 Ultrastructural changes observed in Chlamydomonas reinhardtii, Nannochloropsis oceanica and Phaeodactylum tricornutum following nitrogen starvation. Images were taken from (Fan et al. 2011; Vieler et al. 2012b; Yang et al. 2013), respectively. Rights for reproduction were obtained. $N$ nucleus, $V$ vacuole, $L D$ lipid droplet, Thy thylakoid, $P$ plastoglobule, $C h l$ chloroplast, $P g$ plastoglobule, $O B$ oil bodies

species present in isolated LDs) remains to be determined, although the whole cell lipidome have been reported for $C$. reinhardtii and also for Nannochloropsis oceanica by LCMS/MS based techniques (Liu et al. 2013; Nguyen et al. 2013). LC-MS/MS assisted lipidomic analysis of isolated LDs will reveal content and composition of lipid molecular species present in the lipid bodies, by comparison to the whole cell lipidome, useful insights on the origin and biosynthesis of these molecules in an algal cell should be deduced. Furthermore, Horn et al. (Horn et al. 2012) have elegantly generated comprehensive lipidomic images of the embryo of upper land cotton using a matrix-assisted laser desorption/ionization-MS imaging (MALDI-MSI) approach; application of this technique to a microalgal cell should help map and define the differences in lipid composition and distribution in a subcellular context.

The phospholipid monolayer of LD differs in its composition from other cellular membranes such as endoplasmic reticulum (ER), plasma membrane, or mitochondria. This is consistent with the general view that all membranes are unique in its composition (van Meer et al. 2008). Lipid class analyses by thin layer chromatography have revealed Nevertheless, the LD lipidome (i.e., the lipid molecular 
Table 1 A summary of major proteins present in purified LDs from microalgal species as compared to plants, animals, yeasts and prokaryotes

\begin{tabular}{|c|c|c|c|c|c|}
\hline Organisms & & Major proteins & $\begin{array}{l}\text { Total number } \\
\text { of distinct } \\
\text { proteins } \\
\text { identified }\end{array}$ & LD size & References \\
\hline \multirow[t]{5}{*}{ Microalgae } & Chlamydomonas reinhardtii & MLDP & $>100$ & $\sim 1.5 \mu \mathrm{m}$ & $\begin{array}{l}\text { James et al. (2011), } \\
\text { Moellering and Benning } \\
\text { (2010), Nguyen et al. } \\
\text { (2011) }\end{array}$ \\
\hline & Haematococcus pluvialis & HOGP & na & & Peled et al. (2011) \\
\hline & Dunaliella salina & MLDP & na & & Davidi et al. (2012) \\
\hline & Chlorella sp. & A putative caleosin & na & $>3 \mu \mathrm{m}$ & Lin et al. (2012) \\
\hline & $\begin{array}{l}\text { Nannochloropsis oceanica } \\
\text { (include other species) }\end{array}$ & LDSP & na & & Vieler et al. (2012a) \\
\hline Bryophytes & Physcomitrella patens & Oleosins & na & $0.5-5 \mu \mathrm{m}$ & Huang et al. (2009) \\
\hline \multirow[t]{3}{*}{$\begin{array}{l}\text { Higher } \\
\text { plants }\end{array}$} & Seeds/pollen/tapetum & Oleosins, caleosins, and steroleosins & $>$ a dozen & $0.6-2 \mu \mathrm{m}$ & $\begin{array}{l}\text { Jolivet et al. (2009), } \\
\text { Murphy (1993), Tzen and } \\
\text { Huang (1992) }\end{array}$ \\
\hline & $\begin{array}{l}\text { Oil-rich fruit tissues } \\
\text { (avocado, oil palm) }\end{array}$ & LDAP1 and LDAP2 & around 70 & $10-20 \mu \mathrm{m}$ & Horn et al. (2013) \\
\hline & $\begin{array}{l}\text { Leaf tissue }(>100 \text { plant } \\
\text { species surveyed); } \\
\text { Arabidopsis senescencing } \\
\text { leaves }\end{array}$ & CLO3 and $\alpha$-DOX1 & $>10$ & $1-18 \mu \mathrm{m}$ & $\begin{array}{l}\text { Lersten et al. (2006), } \\
\text { Shimada et al. (2014) }\end{array}$ \\
\hline Animals & Adipose tissues & Proteins of the PAT family & $>100$ & $2-10 \mu \mathrm{m}$ & Goodman (2008) \\
\hline Yeasts & Oleaginous species & $\begin{array}{l}\text { No major structural proteins, but with } \\
\text { the presence of various enzymes of } \\
\text { lipid metabolism detected }\end{array}$ & $>$ a dozen & $0.5-2 \mu \mathrm{m}$ & $\begin{array}{l}\text { Athenstaedt et al. (2006), } \\
\text { Zweytick et al. (2000) }\end{array}$ \\
\hline Prokaryotes & $\begin{array}{l}\text { TAG and wax ester } \\
\text { accumulating bacteria } \\
\text { species }\end{array}$ & $\begin{array}{l}\text { A putative structural protein } \\
\text { identified (LPD06283) }\end{array}$ & $>100$ & $0.1-3 \mu \mathrm{m}$ & $\begin{array}{l}\text { Chen et al. (2014), } \\
\text { Waltermann et al. (2005) }\end{array}$ \\
\hline
\end{tabular}

CLO3 caleosin 3, $\alpha$-DOX1 $\alpha$-dioxygenase, HOGP Haematococcus oil globule protein, LDSP lipid droplet surface protein, LDAP lipid-droplet associated proteins, $M L D P$ major lipid droplet protein, $P A T$ perilipins, adipose-differentiation related protein and tail-interacting protein of $47 \mathrm{kDa}$, na not available

that the betaine lipid diacylglycerol $N^{\prime} N^{\prime} N^{\prime}$-trimethyl homoserine (DGTS) and sulfoquinovosyldiacylglycerol (SQDG) are the two prominent lipid classes associated with isolated LDs from C. reinhardtii (Wang et al. 2009). The high abundance of DGTS as a major polar lipid of lipid droplet isolated from $C$. reinhardtii is consistent with the fact that this algal species lacks phosphatidylcholine (PtdCho), and DGTS is thought to functionally replace it (Liu and Benning 2013). This is also in line with the fact that the betaine lipid synthase (BTA1) is one of the most abundant proteins found in the LD proteome (Moellering and Benning 2010; Nguyen et al. 2011). Membrane lipids present in LDs have also been analyzed for another Chlorophyta alga Haematococcus pluvialis where DGTS, DGDG, PtdCho, and SQDG are the major components (Peled et al. 2011). Both DGTS and PtdCho are present in the Haematococcus LD lipidome; compared to the whole cell lysate, DGTS showed slight enrichment in the LD fraction whereas the content of PtdCho remained similar. The most known function for the presence of these polar lipids in a
LD is their role in forming the monolayer membrane interface to delineate TAGs from an otherwise aqueous subcellular environment. Beyond its structural role, the PLs present in the LDs could also be used as substrates to supply acyl-chains for TAG synthesis.

With the exception of PtdCho (or its homolog DGTS), the overall polar lipid class present in LDs isolated from microalgal species showed some differences to what have been reported previously for higher plants, where phosphoethanolamine (PtdEtn) is often found as the second most abundant polar lipids present in the isolated LDs, where no or only trace level of SQDG or DGDG is found (Katavic et al. 2006; Penno et al. 2013).

Protein composition

\section{Most abundant proteins}

The first proteomics analysis of LDs isolated from microalgae was performed independently by several groups in 
Table 2 Some examples of major composition of isolated lipid droplets na not available

\begin{tabular}{llllll}
\hline & $\begin{array}{l}\text { Mammalian } \\
\text { cells }\end{array}$ & $\begin{array}{l}\text { Plant } \\
\text { seeds }\end{array}$ & Yeasts & A diatom & $\begin{array}{l}\text { Chlamydomonas } \\
\text { reinhardtii }\end{array}$ \\
\hline Protein & $4.8 \%$ & $1.4 \%$ & $2.6 \%$ & $2.3 \%$ & n.a. \\
TAGs & $90.2 \%$ & $95 \%$ & $51.2 \%$ & $58 \%$ & $85-95 \%$ \\
PLs & $4.5 \%$ & $0.9 \%$ & $1.3 \%$ & & $<5 \%$ \\
$\begin{array}{l}\text { Other (free fatty acid, } \beta- \\
\text { carotene, sterol esters) }\end{array}$ & $1.62 \%$ & $4 \%$ & $45 \%$ & na & $10 \%$ FFA \\
References & $\begin{array}{l}\text { Christiansen and } \\
\text { Jensen (1972) }\end{array}$ & $\begin{array}{c}\text { Tzen } \\
\text { et al. } \\
(1993)\end{array}$ & $\begin{array}{c}\text { Leber } \\
\text { et al. } \\
(1994)\end{array}$ & $\begin{array}{c}\text { Nojima } \\
\text { et al. } \\
(2013)\end{array}$ & $\begin{array}{c}\text { Nguyen et al. (2011); } \\
\text { Wang et al. (2009) }\end{array}$ \\
& & & &
\end{tabular}

the model C. reinhardtii. Most of these studies identified the presence of a novel protein named as MLDP (stands for 'Major Lipid Droplet Protein') (James et al. 2011; Moellering and Benning 2010; Nguyen et al. 2011). MLDP has a molecular mass of $28 \mathrm{kDa}$ and is the most abundant protein based on SDS-PAGE separation of total proteins extracted from purified LDs, and has also shown the highest spectra count in LC-MS/MS analyses (Moellering and Benning 2010; Nguyen et al. 2011). Based on primary amino acid sequences, MLDP is predicted as a very hydrophobic protein with no known function domains identified. It shares no primary sequence similarity to known LD proteins including that of plant oleosins (Huang 1992) nor to that of mammalian perilipins (Londos et al. 1999).

A protein homologous to MLDP has also been found as the major protein covering the LDs of Dunaliella salina and was further localized to the surface of LDs by immunogold labelling where hybridized gold particles were found outlining the external circle of lipid droplet (Davidi et al. 2012). However, based on immunofluorescence confocal laser scanning microscopy with anti-MLDP antibodies in C. reinhardtii, Huang et al. (Huang et al. 2013) recently observed MLDP in numerous cup-shaped structures often only partially wrapped around lipid droplets. In this study, MLDP was not found as surrounding lipid droplets as would have been expected for a major structural protein, which was interpreted by the fact that MLDP is localized to subdomains of ER, and only intermittently interacts with the LD surface (Huang et al. 2013).

Protein sequence analyses revealed that orthologs of MLDPs are present in different microalgal species including Volvox carteri, Haematococcus pluvialis, Dunaliella salina, Coccomyxa sp C-169, Chlorella variabilis, Polytomella parva, Prototheca wickerhamii and Micromonas pusilla CCMP1545. However, no ortholog has been identified so far in diatoms, red algae, bryophytes or higher plants. Phylogenetic comparison of these different MLDP orthologs have identified two evolutionary groups (Volvocales vs. Chlorellales), in line with the evolution pattern of green lineage (Davidi et al. 2012). In
Haematococcus pluvialis (Peled et al. 2011) and Dunaliella salina (Davidi et al. 2012), the MLDP homologs have been experimentally found to be indeed the major protein of lipid droplets. Interestingly, in some Chlorella species, the major protein associated with lipid droplets was found to be not a MLDP homolog, but a homolog of the minor plant oil-body protein called caleosin (Katavic et al. 2006; Lin et al. 2012).

Proteomic studies of the LDs isolated from the heterokont microalga Nannochloropsis oceanica revealed yet another distinct protein named as Lipid Droplet Surface Protein (LDSP) as the most abundant protein (Vieler et al. 2012a). LDSP has a molecular weight of $17 \mathrm{kDa}$, contained unique primary sequences and with no previously assigned functions. LDSP is present in all six species of Nannochloropsis but no homologs can be found in other heterokont classes, or in the green or animal lineage (Vieler et al. 2012a). This is not surprising since the major proteins identified in the LDs from different phyla are quite distinct and Eustigmatophytes are known to have evolved early from other heterokont classes.

Major lipid-droplet proteins identified so far in microalgae and their characteristics are summarized in Table 1 and compared to LD proteins of plant and mammalian origin. It is worth mentioning that except in $C$. reinhardtii where extensive proteomic analysis was carried out (Moellering and Benning 2010; Nguyen et al. 2011), later studies performed on other species have mostly focused on mass spectrometry identification of the major protein present in the isolated lipid droplets, thus the entire LD proteome remains to be determined in these species.

Despite differences in their primary amino acid sequences, some commonalities do exist among all known major LD proteins: (1) They are all highly hydrophobic proteins despite their distinct primary sequences; (2) The expression of genes encoding these proteins is limited to conditions favoring oil synthesis (Davidi et al. 2012; Moellering and Benning 2010; Vieler et al. 2012a); and (3) Similar to what has been observed for oleosins (Siloto et al. 2006), repression of MLDP gene expression in 
Chlamydomonas increased the size of lipid droplets, thus supporting the general view that major LD protein prevents fusion and maintains LD size (Moellering and Benning 2010). Other functions beyond a merely structural one for these most abundant LD proteins (i.e., MLDP, or LDSP) in microalgae remain to be investigated. For example, one study described one isoform of oleosin isolated from Arachis hypogaea as a bifunctional enzyme with both monoacylglycerol acyltransferase and a phospholipase A(2) activities (Parthibane et al. 2012).

\section{Lipid-related proteins}

The development of advanced proteomics tools allowed the identification of not only the most abundant but also minor proteins present in LDs. For example, $>200$ proteins were identified in purified lipid droplets of $C$. reinhardtii (Moellering and Benning 2010; Nguyen et al. 2011), a feature contrary to oil bodies of plant oilseeds where relatively fewer proteins have been detected (Jolivet et al. 2009), but strikingly similar to the proteome of mammalian lipid droplets (Farese and Walther 2009; Goodman 2008). This particular protein composition of algal lipid droplets could reflect the fact that $C$. reinhardtii is derived from a common ancestor of plants and animals over 1 billion years ago, and some features have been conserved along one lineage, whereas others lost along the other lineage (Merchant et al. 2007).

Besides MLDP, the lipid droplet proteome of Chlamydomonas is mainly made of proteins involved in metabolism, transport, vesicle trafficking, redox and other functions. Among all proteins, $>10 \%$ were related to lipid metabolism; this ratio is increased to $35 \%$ when only the top 20 proteins showing the highest spectra count is considered. Considering that Chlamydomonas genome contains $\sim 15,000$ genes (Merchant et al. 2007), if we estimate that around 100 proteins are involved in TAG metabolism based on what we know from the plant model Arabidopsis thaliana (Li-Beisson et al. 2010), this means that at least $0.6 \%$ Chlamydomonas genes could be related to TAG synthesis or turnover. This support the notion that proteome of lipid droplet is highly enriched in proteins of lipid metabolism.

Lipid-related proteins identified in the purified lipid droplets in $C$. reinhardtii include acyl activating enzymes, lipases, lipid trafficking proteins, acyltransferases of the Kennedy pathway [i.e., a glycerol-3-phosphate acyltransferase (GPAT), a lysophosphatidic acid acyltransferase (LPAT)], enzymes of the transacylation pathway [the phospholipid:diacylglycerol acyltransferase (PDAT) and two lysophosphatidyl lipid acyltransferases (LPLAT)], and also membrane lipid synthesizing enzymes, for example, the betaine lipid synthase (BTA1) responsible for synthesis of DGTS (Moellering and Benning 2010; Nguyen et al. 2011).
DGTS synthase was identified as possessing the third highest spectral count following MLDP and an $\alpha / \beta$ hydrolase (Chlre4/330619, eq. to Cre12.g540550). The presence of high amount of BTA1 is consistent with the biochemical finding that DGTS is one of the major membrane lipids covering the TAG core. This is also in synergy with what have been found in other model organisms, where PtdCho is the major polar lipids surrounding the droplet, and the PtdCho synthesizing enzyme CTP:phosphocholine cytidyltransferase (CCT) is present in the LD proteome (Guo et al. 2008). The presence of both neutral and polar lipid synthesizing enzymes point out the essential metabolic co-ordination that is required when lipid droplets are formed in response to particular cellular needs.

As well as lipid synthesizing enzymes, hydrolases/lipases, etc,. are one of the major classes, i.e., $>20 \%$ of all lipid-related proteins present in the proteome of lipid droplet are potentially lipid-hydrolyzing enzymes. Lipases catalyze lipid hydrolysis, releasing free fatty acids and glycerol. Presence of these proteins in LDs is consistent with its role in ensuring TAG remobilization and LD turnover. Indeed, in the yeast Saccharomyces cerevisiae (Athenstaedt and Daum 2005) and in the higher plant Arabidopsis thaliana (Eastmond 2006), TAG lipase activities have been found to be associated with LDs, to which these proteins have further been localized using protein fusion techniques.

A number of known plastidial-lipid trafficking proteins (Benning 2008) are also present in the proteome of lipid droplets. The presence of these presumably chloroplast envelop proteins in the LD proteome supports the current view that plastid envelops are important in the formation of Chlamydomonas lipid droplet (Goodson et al. 2011). This is also in line with recent data from higher plant models where transorganellar complementation studies have led to the proposition that hemi-membrane fusion at the plastid and ER contact site facilitate inter-organellar interactions and allow enzymes direct access to substrates present in both organelle membranes (Mehrshahi et al. 2013, 2014). In Chlamydomonas reinhardtii, the observation that one single plastidial $\omega-3$ fatty acid desaturase can act on the substrates present in ER and plastid compartment further supports such a view (Mehrshahi et al. 2014; Nguyen et al. 2013).

\section{Vesicle trafficking and others}

In addition to lipid-related proteins, a considerable number of proteins potentially involved in vesicle trafficking and transport has been identified in LD proteomes. One of the major classes of proteins present is the small Rab-type GTPases. Some members of this family are also present in the LD proteome of other organisms and have been shown to be involved in the membrane trafficking and inter- 
organellar communications (Martin and Parton 2008; Murphy et al. 2009). For example, RNAi silencing of an Arf79a protein, a member of the GTPases, has revealed its potential involvement in LD formation and utilization in Drosophila $S 2$ cells (Guo et al. 2008). The presence of membrane-traffic proteins in LDs gives support that LDs are dynamic structures specialized in inter-membrane lipid transport. The presence of putative GTPase orthologs in $C$. reinhardtii suggested a similar function of lipid droplets in membrane trafficking. Recently, a link between vesicle trafficking and lipid remobilization has been proposed based on the studies of the effect of Brefeldin A in Chlamydomonas cells (Kato et al. 2013).

The presence of large number of photosystem proteins indicates potential contamination during purification procedure (Moellering and Benning 2010; Nguyen et al. 2011), and could also indicate genuine associations between these subcellular organelles as increasing evidence indicates the importance of chloroplast and chloroplast envelop in LD biogenesis (discussed more in detail in the biogenesis section). As with most proteomic studies of a similar nature, experimental demonstration of a 'true' association of these proteins to LD remains to be tested. Dynamic turnover of LD proteome in response to environmental conditions is not known, and research in this direction should yield important insight in understanding the formation and functions of this fascinating organelle.

So far, the largest group within the proteome of isolated LDs falls into the functional category of 'unknown'. With increasing technological development combined with gene discoveries, these 'unknown' proteins might be assigned new functions, thus pointing toward yet even wider cellular functions that are associated with or has been accredited to lipid droplets. It is worth noting that current proteomic studies report only most tightly associated proteins; thus, proteins that are loosely attached to lipid droplets might have been overlooked. These loosely associated proteins could also be important in the function and biogenesis of the lipid droplets.

\section{Biogenesis of lipid droplets}

In plants, yeasts and mammalian cells, it is generally accepted that LDs are largely present in the cytosol and formed via extension of specific domains of ER (i.e., budding model) (Huang 1992; Murphy 2001). A cytosolic location also allows potential interactions with other subcellular organelles including ER, mitochondria, and peroxisomes (Chapman et al. 2012; Farese and Walther 2009; Goodman 2008). Recently, two studies suggested the involvement of plastids in the formation of LDs in $C$. reinhardtii (Fan et al. 2011; Goodson et al. 2011); and in particular, LDs were observed in both plastid and cytosol in a starch-less mutant of C. reinhardtii (Fan et al. 2011). Based on these studies, a possible model of LD biogenesis involving both ER and plastid envelops is proposed and shown in Fig. 2.

The participation of plastids in LD biogenesis is further supported by a likely plastidial location of TAG-synthesizing enzymes in Chlamydomonas as predicted by an algorithm specially designed for predicting targeting signals of microalgal proteins (Tardif et al. 2012). TAG assembly usually shares common pathways with synthesis of membrane lipids until the formation of DAGs, i.e., the well-known Kennedy pathway (Kennedy 1956). In higher plants, two parallel pathways leading to the sequential acylation of a glycerol 3-phosphate (G3P) until the formation of DAGs occur in plastid and also in ER, namely the prokaryotic and the eukaryotic pathway, respectively (Roughan and Slack 1982). These two sets of reactions are driven by GPAT, LPAT, and a phosphatidic acid phosphatase (PAP); isoforms of each enzyme occur in both compartments in Arabidopsis thaliana. However, in Chlamydomonas, the homologs of plant ER-type acyltransferases (GPAT and LPAT) and PAP are found to be single enzymes, and are predicted to possess chloroplasttargeting signals at their N-termini (Tardif et al. 2012).

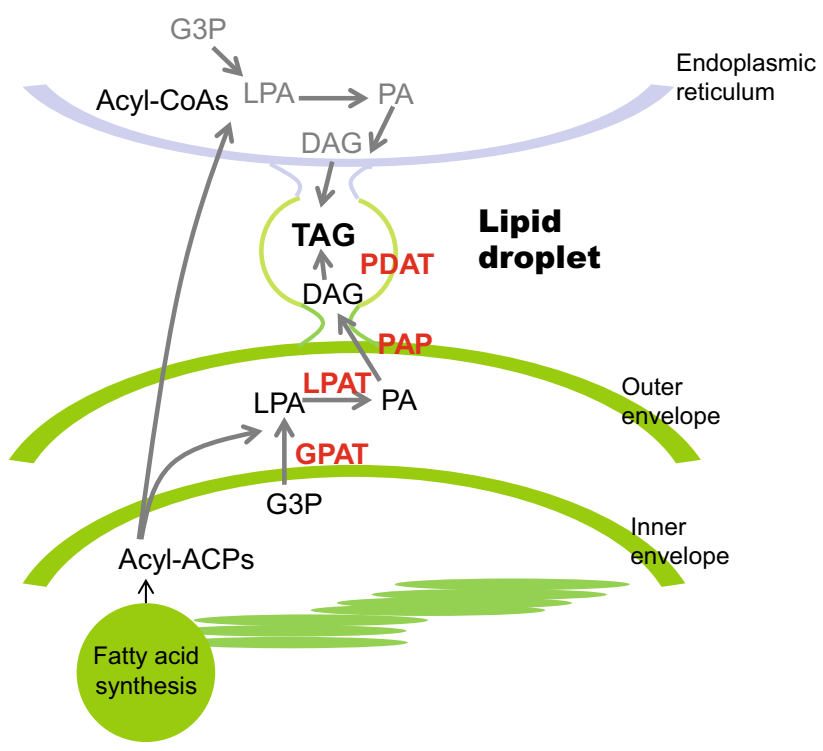

Fig. 2 One of the possible models of LD biogenesis in Chlamydomonas reinhardtii involving both plastid and ER. The TAG-synthesizing proteins present in the LD proteome are highlighted. In red are enzymes; in black are lipid substrates. This model is partly based on that of (Goodson et al. 2011; Nguyen et al. 2011). ACP acyl carrier protein, $C O A$ coenzyme A, $D A G$ diacylglycerol, $G 3 P$ glycerol-3phosphate, GPAT glycerol-3-phosphate acyltransferase, LPA lysophosphatidic acid, LPAT lysophosphatidic acid acyltransferase, $P A$ phosphatidic acid, $P A P$ phosphatidic acid phosphatase, PDAT phospholipid:diacylglycerol acyltransferase, $T A G$ triacylglycerol 
The final step of TAG synthesis is the acylation of the $s n-3$ position of a DAG molecule to form TAG. This acylation reaction can be done via two routes, i.e., acylCoA-dependent and acyl-CoA-independent reactions. The former is catalyzed by diacylglycerol acyltransferase (DGAT), whereas the latter is catalyzed by PDAT, a phospholipid: diacylglycerol acyltransferase. Only 1 PDAT protein is present in Chlamydomonas (Yoon et al. 2012), whereas six genes (DGAT1; DGTT1-5) encoding diacylglycerol acyltransferases (DGAT) are encoded in the genome of Chlamydomonas (Boyle et al. 2012), among which DGTT1 (g1030.t1) has been shown to be essential for TAG accumulation under $-\mathrm{N}$ stress and this protein is also predicted to have an $\mathrm{N}$-terminal chloroplast transit peptide. Based on primary sequences, Chlamydomonas PDAT also possess an N-terminal chloroplast-targeting sequence of $\sim 65$ amino acids. The demonstration that PDAT can use galactolipids in vitro as substrates supports such a location (Yoon et al. 2012).

It is worth mentioning that above subcellular predictions do not rule out the possibility of a dual localization for any of these enzymes. Nevertheless, these analyses of TAG-synthesizing enzymes and their likely subcellular locations suggest that the whole pathway from G3P to TAG could involve the plastid, which is consistent with the plastidial origin of TAG backbones (Fan et al. 2011). A plastidial location of TAG-synthesizing pathway in a microalga has implications not only for our overall understanding of the subcellular organization of glycerolipid metabolism and turnover, but also has significant impact on biotechnological applications. For example, the plastid is already a platform for production of high value proteins (Bock 2001; Specht et al. 2010), and the possibility that an entire TAG-synthesizing pathway could be present in the plastid would allow us to engineer the plastid genome of $C$. reinhardtii to improve oil yields and composition, and open up possibilities of using lipid droplets as a destination for storage of other hydrophobic molecules.

Moreover, these lipid synthesizing enzymes can also play an active role in LD biogenesis. For example, it has been recently demonstrated that a mutant (pah1 $\Delta$ ) of Saccharomyces cerevisiae defective in the lipin phosphatidic acid phosphohydrolase (PAH1) possessed $>60 \%$ less of the total number of lipid droplets, whereas the mutant cells still synthesized equal amount of neutral lipids (Adeyo et al. 2011). The authors conclude that PAH1 plays a role in LD biogenesis through modulation of the intracellular level of DAGs. Chlamydomonas encodes one putative homolog of PAH1 (Cre12.g506600, Phytozome version 10). It will be worthwhile to test if and to what extent Cre12.g506600 plays in LD biogenesis in an algal cell.

\section{Functions of lipid droplets in microalgae}

LDs are made in response to particular cellular needs. The number of lipid droplets per cell varies depending on cell type, developmental stages and growth conditions. Increasing knowledge of lipid droplets has revealed other functions beyond energy storage (Fig. 3). Below, we summarize demonstrated and inferred functions attributed to lipid droplets.

Storage of carbon and energy under adverse conditions

TAGs contain twice more energy than starch and protein per weight unit; thus, TAG storage is the most efficient and compact way to pack cellular energies. Carbon and energy storage in the form of triacylglycerols seem to be a unifying nature of all eukaryotic cells. In microalgae, when nutrient is not available, cells store extra carbon as TAGs, thus prepared for long-term usage ( $\mathrm{Hu}$ et al. 2008). Enhanced fatty acid synthesis and their storage as TAGs under nutrient depletion also provide an additional electron sink because acyl groups are among the most reduced carbon compounds. This capacity in sequestering of extra electrons is especially important when cells were cultivated under nutrient deplete conditions, when the metabolic energetic need is reduced, i.e., cessation of cell growth.

Oil accumulation as a major survival strategy has recently been demonstrated through the study of a Chlamydomonas mutant pgdl (stands for plastid galactoglycerolipid degradation 1) (Li et al. 2012a). The pgdl mutant accumulated twice less oil than its wild-type background, and also exhibited a loss of viability under nitrogendeprived conditions. In vitro biochemical and substrate labelling studies have demonstrated that PGD1 encodes a galactoglycerolipid lipase, which is important for channeling fatty acids from galactolipids to TAG following nitrogen starvation. Detailed physiological characterization of the pgdl mutant provided evidence that TAGs

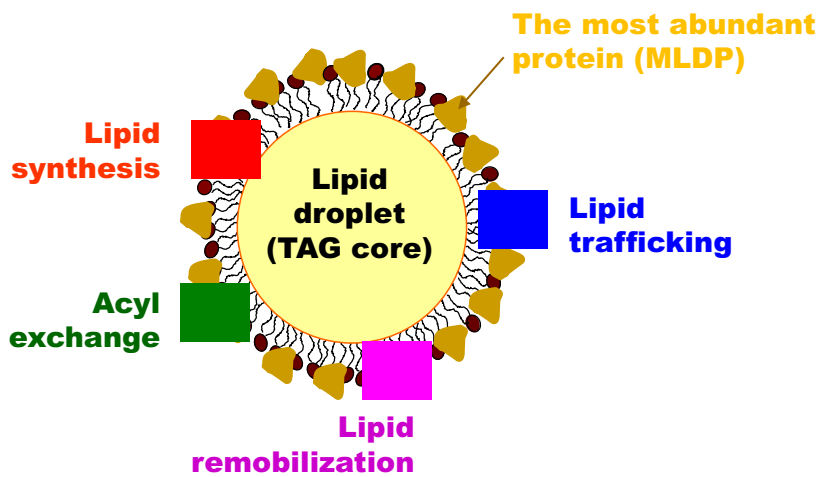

Fig. 3 LDs are at the heart of cellular lipid homeostasis in Chlamydomonas reinhardtii 
synthesized following nitrogen deprivation relieve an overproduction of photosynthetic electron transport chains, thus ensuring cell survival under these conditions.

A depot of acyl chains for membrane synthesis

TAG accumulation and appearance of lipid droplets were also observed under conditions where no net synthesis of fatty acids was observed, for example, under iron starvation in algae (Urzica et al. 2013). This supports the view that LDs serve as a temporary depot for acyl chains. Under adverse conditions (freezing, osmotic stress, etc.), cells developed sophisticated mechanisms to remove certain membrane lipids to compensate for changes in bilayer structures; and these released acyl chains can be assembled to form TAGs contained in LDs. When environmental conditions become favorable again, the acyl chains are released from TAGs through activities of TAG hydrolyzing enzymes, and used for membrane lipid synthesis and assembly. This mechanism provides efficient means for cells to assemble their membrane lipids quickly, thus bypassing the need for de novo fatty acid synthesis which could apparently require more biochemical steps and hence takes more time. LDs thus serve as a central carrier for correct shuffling of acyl chains between different lipid classes thus important in ensuring overall cellular lipid homeostasis.

\section{Other functions}

Besides as a storage depot of acyl chains for membrane synthesis and energy supply, microalgal lipid droplets are postulated to play numerous roles in cellular physiology. These roles are hypothesized based on the identification of a large array of proteins in the LD proteome (Moellering and Benning 2010; Nguyen et al. 2011), as well as some evidence in other organisms. These roles include cell signaling, protective roles, acting as a cargo for delivery of compounds to their subcellular location, a reservoir for some compounds to avoid detrimental effect on normal cellular functions, etc. Experimental demonstration of the many postulated roles of LDs in microalgae is, however, hampered by the lack of mutants impaired in LD biogenesis and metabolism.

Concluding remarks and future directions

Over the past few years, rapid progress has been made toward the structural, function and biogenesis of LDs isolated from the model microalga $C$. reinhardtii. Nevertheless, a number of important questions remain unanswered. Firstly, modern LC-MS/MS based proteomics allowed the identification of hundreds of proteins associated to the isolated LDs; functional characterization and cell biological evidence for a 'genuine' association of these proteins to the lipid droplet remain to be demonstrated. Secondly, most current studies focused on LDs isolated from $-\mathrm{N}$ stress condition; what are the proteins and lipids associated with LDs formed under other conditions? Thirdly, what is the organization and functions of polar lipids around the LD; do they form functional subdomains or are they evenly distributed across the LD surface? And finally, what are the regulatory mechanisms that control the number and size of lipid droplet? If under transcriptional regulation, then what are the identity and networks of transcriptional factors in microalgae? These and other unanswered questions should provide a wealth of research opportunities related to the study of basic and applied questions in algal LD biology and biotechnology.

Author contribution statement H. Goold, F. Beisson, G. Peltier, Y. Li-Beisson all participated the writing of the manuscript.

Acknowledgments Work in the authors' laboratory is supported by the French Agence Nationale pour la Recherche (ANR-Diesalg: ANR-12-BIME-0001-02 and ANR-MUsCA: ANR-13-JSV5-0005). Hugh Goold acknowledges The University of Sydney for a $\mathrm{PhD}$ studentship.

Conflict of interest The authors declare that they have no conflict of interest.

\section{References}

Adeyo O, Horn PJ, Lee SK, Binns DD, Chandrahas A, Chapman KD, Goodman JM (2011) The yeast lipin orthologue Pah1p is important for biogenesis of lipid droplets. J Cell Biol 192:1043-1055. doi:10.1083/jcb.201010111

Athenstaedt K, Daum G (2005) Tgl4p and Tgl5p, two triacylglycerol lipases of the yeast Saccharomyces cerevisiae are localized to lipid particles. J Biol Chem 280:37301-37309. doi:10.1074/jbc. M507261200

Athenstaedt K, Jolivet P, Boulard C, Zivy M, Negroni L, Nicaud J-M, Chardot T (2006) Lipid particle composition of the yeast Yarrowia lipolytica depends on the carbon source. Proteomics 6:1450-1459. doi:10.1002/pmic.200500339

Benning C (2008) A role for lipid trafficking in chloroplast biogenesis. Prog Lipid Res 47:381-389

Bock R (2001) Transgenic plastids in basic research and plant biotechnology. J Mol Biol 312:425-438. doi:10.1006/jmbi.2001. 4960

Bourgis F, Kilaru A, Cao X, Ngando-Ebongue GF, Drira N, Ohlrogge JB, Arondel V (2011) Comparative transcriptome and metabolite analysis of oil palm and date palm mesocarp that differ dramatically in carbon partitioning. Proc Natl Acad Sci USA 108:12527-12532. doi:10.1073/pnas.1106502108

Boyle NR et al (2012) Three acyltransferases and nitrogen-responsive regulator are implicated in nitrogen starvation-induced triacylglycerol accumulation in Chlamydomonas. J Biol Chem 287:15811-15825. doi:10.1074/jbc.M111.334052

Cagnon C et al (2013) Development of a forward genetic screen to isolate oil mutants in the green microalga Chlamydomonas reinhardtii. Biotechnol Biofuels 6:178 
Chapman KD, Dyer JM, Mullen RT (2012) Biogenesis and functions of lipid droplets in plants: thematic review series: lipid droplet synthesis and metabolism: from yeast to man. J Lipid Res 53:215-226

Chen $\mathrm{Y}$ et al (2014) Integrated omics study delineates the dynamics of lipid droplets in Rhodococcus opacus PD630. Nucleic Acids Res 42:1052-1064. doi:10.1093/nar/gkt932

Christiansen K, Jensen PK (1972) Membrane-bound lipid particles from beef heart chemical composition and structure. BBA-Lipid Lipid Met 260:449-459. doi:10.1016/0005-2760(72)90060-4

Czabany T, Athenstaedt K, Daum G (2007) Synthesis, storage and degradation of neutral lipids in yeast. BBA-Mol Cell Biol Lipids 1771:299-309. doi:10.1016/j.bbalip.2006.07.001

Czabany T, Wagner A, Zweytick D, Lohner K, Leitner E, Ingolic E, Daum G (2008) Structural and biochemical properties of lipid particles from the yeast Saccharomyces cerevisiae. J Biol Chem 283:17065-17074. doi:10.1074/jbc.M800401200

Davidi L, Katz A, Pick U (2012) Characterization of major lipid droplet proteins from Dunaliella. Planta 236:19-33. doi:10.1007/ s00425-011-1585-7

Eastmond PJ (2006) SUGAR-DEPENDENT1 encodes a patatin domain triacylglycerol lipase that initiates storage oil breakdown in germinating Arabidopsis seeds. Plant Cell 18:665-675. doi:10. $1105 /$ tpc. 105.040543

Fan JL, Andre C, Xu CC (2011) A chloroplast pathway for the de novo biosynthesis of triacylglycerol in Chlamydomonas reinhardtii. FEBS Lett 585:1985-1991. doi:10.1016/j.febslet.2011.05.018

Farese RV Jr, Walther TC (2009) Lipid droplets finally get a little R-E-S-P-E-C-T. Cell 139:855-860

Goodman JM (2008) The gregarious lipid droplet. J Biol Chem 283:28005-28009. doi:10.1074/jbc.R800042200

Goodson C, Roth R, Wang ZT, Goodenough U (2011) Structural correlates of cytoplasmic and chloroplast lipid body synthesis in Chlamydomonas reinhardtii and stimulation of lipid body production with acetate boost. Eukaryot Cell 10:1592-1606. doi:10.1128/ec.05242-11

Graham IA (2008) Seed storage oil mobilization. Annu Rev Plant Biol 59:115-142. doi:10.1146/annurev.arplant.59.032607.092938

Greenspan P, Mayer E, Fowler S (1985) Nile red: a selective fluorescent stain for intracellular lipid droplets. J Cell Biol 100:965-973

Guo Y et al (2008) Functional genomic screen reveals genes involved in lipid-droplet formation and utilization. Nature 453:657-661. doi:10.1038/nature06928

Hemschemeier A, Casero D, Liu B, Benning C, Pellegrini M, Happe T, Merchant SS (2013) COPPER RESPONSE REGULATOR1dependent and-independent responses of the Chlamydomonas reinhardtii transcriptome to dark anoxia. Plant Cell 25:3186-3211. doi:10.1105/tpc. 113.115741

Horn PJ et al (2012) Spatial mapping of lipids at cellular resolution in embryos of cotton. Plant Cell 24:622-636. doi:10.1105/tpc.111. 094581

Horn PJ et al (2013) Identification of a new class of lipid dropletassociated proteins in plants. Plant Physiol 162:1926-1936. doi: $10.1104 /$ pp. 113.222455

Hu Q, Sommerfeld M, Jarvis E, Ghirardi M, Posewitz M, Seibert M, Darzins A (2008) Microalgal triacylglycerols as feedstocks for biofuel production: perspectives and advances. Plant J 54:621-639

Huang AHC (1992) Oil bodies and oleosins in seeds. Annu Rev Plant Physiol Plant Molec Biol 43:177-200

Huang AHC (1996) Oleosins and oil bodies in seeds and other organs. Plant Physiol 110:1055-1061. doi:10.1104/pp.110.4.1055

Huang C-Y, Chung C-I, Lin Y-C, Hsing Y-IC, Huang AHC (2009) Oil bodies and oleosins in Physcomitrella possess characteristics representative of early trends in evolution. Plant Physiol 150:1192-1203. doi:10.1104/pp.109.138123
Huang N-L, Huang M-D, Chen T-LL, Huang AHC (2013) Oleosin of subcellular lipid droplets evolved in green algae. Plant Physiol 161:1862-1874. doi:10.1104/pp.112.212514

James GO, Hocart CH, Hillier W, Chen H, Kordbacheh F, Price GD, Djordjevic MA (2011) Fatty acid profiling of Chlamydomonas reinhardtii under nitrogen deprivation. Bioresour Technol 102:3343-3351

Jolivet P et al (2009) Protein composition of oil bodies from mature Brassica napus seeds. Proteomics 9:3268-3284. doi:10.1002/ pmic. 200800449

Katavic V, Agrawal GK, Hajduch M, Harris SL, Thelen JJ (2006) Protein and lipid composition analysis of oil bodies from two Brassica napus cultivars. Proteomics 6:4586-4598. doi:10.1002/ pmic. 200600020

Kato N, Dong T, Bailey M, Lum T, Ingram D (2013) Triacylglycerol mobilization is suppressed by brefeldin A in Chlamydomonas reinhardtii. Plant Cell Physiol 54:1585-1599. doi:10.1093/pcp/ pct103

Kennedy EP (1956) The biological synthesis of phospholipids. Can J Biochem Phys 34:334-348

Kim S et al (2013) Rapid induction of lipid droplets in Chlamydomonas reinhardtii and Chlorella vulgaris by brefeldin A. PLoS One 8:e81978. doi:10.1371/journal.pone.0081978

Leber R, Zinser E, Paltauf F, Daum G, Zellnig G (1994) Characterization of lipid particles of the yeast, Saccharomyces cerevisiae. Yeast 10:1421-1428. doi:10.1002/yea.320101105

Lersten NR, Czlapinski AR, Curtis JD, Freckmann R, Horner HT (2006) Oil bodies in leaf mesophyll cells of angiosperms: overview and a selected survey. Am J Bot 93:1731-1739. doi:10. 3732/ajb.93.12.1731

Li X et al (2012a) A galactoglycerolipid lipase Is required for triacylglycerol accumulation and survival following nitrogen deprivation in Chlamydomonas reinhardtii. Plant Cell 24:4670-4686. doi:10.1105/tpc.112.105106

Li XB, Benning C, Kuo MH (2012b) Rapid triacylglycerol turnover in Chlamydomonas reinhardtii requires a lipase with broad substrate specificity. Eukaryot Cell 11:1451-1462. doi:10.1128/ec. 00268-12

Li-Beisson Y, Shorrosh B, Beisson F, Andersson M, Arondel V, Bates P, Baud S, Bird D, DeBono A, Durrett T, Franke R, Graham I, Katayama K, Kelly A, Larson T, Markham J, Miquel M, Molina I, Nishida I, Rowland O, Samuels L, Schmid K, Wada H, Welti R, Xu C, Zallot R and Ohlrogge J (2010) Acyl lipid metabolism. In: Last R (ed) The Arabidopsis Book 8: e0999, doi:10.1199/tab. 0999

Lin IP, Jiang PL, Chen CS, Tzen JTC (2012) A unique caleosin serving as the major integral protein in oil bodies isolated from Chlorella $s p$ cells cultured with limited nitrogen. Plant Physiol Biochem 61:80-87. doi:10.1016/j.plaphy.2012.09.008

Liu B, Benning C (2013) Lipid metabolism in microalgae distinguishes itself. Curr Opin Biotech 24:300-309. doi:10.1016/j. copbio.2012.08.008

Liu B, Vieler A, Li C, Daniel Jones A, Benning C (2013) Triacylglycerol profiling of microalgae Chlamydomonas reinhardtii and Nannochloropsis oceanica. Bioresour Technol 146:310-316. doi:10.1016/j.biortech.2013.07.088

Londos C, Brasaemle DL, Schultz CJ, Segrest JP, Kimmel AR (1999) Perilipins, ADRP, and other proteins that associate with intracellular neutral lipid droplets in animal cells. Semin Cell Dev Biol 10:51-58

Martin S, Parton RG (2008) Characterization of Rab18, a lipid droplet-associated small GTPase. In: William E, Balch CJD, Alan H (eds) Methods in Enzymology, vol 438. Academic Press, New York, pp 109-129. doi:10.1016/S0076-6879(07)38008-7

Mehrshahi P, Stefano G, Andaloro JM, Brandizzi F, Froehlich JE, DellaPenna D (2013) Transorganellar complementation 
redefines the biochemical continuity of endoplasmic reticulum and chloroplasts. Proc Natl Acad Sci USA 110:12126-12131. doi:10.1073/pnas.1306331110

Mehrshahi P, Johnny C, DellaPenna D (2014) Redefining the metabolic continuity of chloroplasts and ER. Trends Plant Sci 19:501-507. doi:10.1016/j.tplants.2014.02.013

Merchant SS et al (2007) The Chlamydomonas genome reveals the evolution of key animal and plant functions. Science 318:245-250. doi:10.1126/science.1143609

Merchant SS, Kropat J, Liu B, Shaw J, Warakanont J (2012) TAG, You're it! Chlamydomonas as a reference organism for understanding algal triacylglycerol accumulation. Curr Opin Biotech 23:352-363

Miller R et al (2010) Changes in transcript abundance in Chlamydomonas reinhardtii following nitrogen deprivation predict diversion of metabolism. Plant Physiol 154:1737-1752. doi:10.1104/ pp. 110.165159

Moellering ER, Benning C (2010) RNA interference silencing of a major lipid droplet protein affects lipid droplet size in Chlamydomonas reinhardtii. Eukaryot Cell 9:97-106. doi:10.1128/ ec.00203-09

Mou SL et al (2012) Rapid estimation of lipid content in an Antarctic ice alga (Chlamydomonas sp.) using the lipophilic fluorescent dye BODIPY505/515. J Appl Phycol 24:1169-1176. doi:10. 1007/s10811-011-9746-4

Murphy DJ (1993) Structure, function and biogenesis of storage lipid bodies and oleosins in plants. Prog Lipid Res 32:247-280

Murphy DJ (2001) The biogenesis and functions of lipid bodies in animals, plants and microorganisms. Prog Lipid Res 40:325-438. doi:10.1016/S0163-7827(01)00013-3

Murphy S, Martin S, Parton RG (2009) Lipid droplet-organelle interactions; sharing the fats. BBA-Mol Cell Biol L 1791:441-447. doi:10.1016/j.bbalip.2008.07.004

Nguyen HM et al (2011) Proteomic profiling of oil bodies isolated from the unicellular green microalga Chlamydomonas reinhardtii: With focus on proteins involved in lipid metabolism. Proteomics 11:4266-4273. doi:10.1002/pmic.201100114

Nguyen HM et al (2013) The green microalga Chlamydomonas reinhardtii has a single $\omega-3$ fatty acid desaturase that localizes to the chloroplast and impacts both plastidic and extraplastidic membrane lipids. Plant Physiol 163:914-928. doi:10.1104/pp.113.223941

Nojima D, Yoshino T, Maeda Y, Tanaka M, Nemoto M, Tanaka T (2013) Proteomics analysis of oil body-associated proteins in the oleaginous diatom. J Proteome Res 12:5293-5301. doi:10.1021/ pr4004085

Parthibane V, Rajakumari S, Venkateshwari V, Iyappan R, Rajasekharan R (2012) Oleosin is bifunctional enzyme that has both monoacylglycerol acyltransferase and phospholipase activities. J Biol Chem 287:1946-1954. doi:10.1074/jbc.M111.309955

Peled E, Leu S, Zarka A, Weiss M, Pick U, Khozin-Goldberg I, Boussiba $S$ (2011) Isolation of a novel oil globule protein from the green alga Haematococcus pluvialis (Chlorophyceae). Lipids 46:851-861. doi:10.1007/s11745-011-3579-4

Penno A, Hackenbroich G, Thiele C (2013) Phospholipids and lipid droplets. BBA-Mol Cell Biol L 1831:589-594. doi:10.1016/j. bbalip.2012.12.001

Roughan PG, Slack CR (1982) Cellular organization of glycerolipid metabolism. Ann Rev Plant Physio 33:97-132. doi:10.1146/ annurev.pp.33.060182.000525

Shimada TL et al (2014) Leaf oil body functions as a subcellular factory for the production of a phytoalexin in Arabidopsis. Plant Physiol 164:105-118. doi:10.1104/pp.113.230185

Siaut $M$ et al (2011) Oil accumulation in the model green alga Chlamydomonas reinhardtii: characterization, variability between common laboratory strains and relationship with starch reserves. BMC Biotech 11:7
Siloto RMP, Findlay K, Lopez-Villalobos A, Yeung EC, Nykiforuk CL, Moloney MM (2006) The accumulation of oleosins determines the size of seed oilbodies in Arabidopsis. Plant Cell 18:1961-1974. doi:10.1105/tpc.106.041269

Specht E, Miyake-Stoner S, Mayfield S (2010) Micro-algae come of age as a platform for recombinant protein production. Biotech Let 32:1373-1383. doi:10.1007/s10529-010-0326-5

Tardif $M$ et al (2012) PredAlgo, a new subcellular localization prediction tool dedicated to green algae. Mol Biol Evol. doi:10. 1093/molbev/mss 178

Tzen JTC, Huang AHC (1992) Surface structure and properties of plant seed oil bodies. J Cell Biol 117:327-335

Tzen JTC, Cao YZ, Laurent P, Ratnayake C, Huang AHC (1993) Lipids, proteins, and structure of seed oil bodies from divers species. Plant Physiol 101:267-276

Urzica EI et al (2013) Remodeling of membrane lipids in iron-starved Chlamydomonas. J Biol Chem 288:30246-30258. doi:10.1074/ jbc.M113.490425

van Meer G, Voelker DR, Feigenson GW (2008) Membrane lipids: where they are and how they behave. Nat Rev Mol Cell Biol 9:112-124. doi: $10.1038 / \mathrm{nrm} 2330$

Vieler A, Brubaker SB, Vick B, Benning C (2012a) A lipid droplet protein of Nannochloropsis with functions partially analogous to plant oleosins. Plant Physiol 158:1562-1569. doi:10.1104/pp. 111.193029

Vieler A et al (2012b) Genome, functional gene annotation, and nuclear transformation of the heterokont oleaginous alga Nannochloropsis oceanica CCMP1779. PLoS Genet 8:e1003064. doi:10.1371/journal.pgen.1003064

Waltermann M et al (2005) Mechanism of lipid-body formation in prokaryotes: how bacteria fatten up. Mol Microbiol 55:750-763. doi:10.1111/j.1365-2958.2004.04441.x

Wang ZT, Ullrich N, Joo S, Waffenschmidt S, Goodenough U (2009) Algal lipid bodies: stress induction, purification, and biochemical characterization in wild-type and starchless Chlamydomonas reinhardtii. Eukaryot Cell 8:1856-1868

Xie B et al (2014) High-throughput fluorescence-activated cell sorting for lipid hyperaccumulating Chlamydomonas reinhardtii mutants. Plant Biotech J 12:872-882. doi:10.1111/pbi.12190

Yan C, Fan J, Xu C (2013) Chapter 5-Analysis of oil droplets in microalgae. In: Hongyuan Y, Peng L (eds) Methods in cell biology, vol 116. Academic Press, New York, pp 71-82. doi:10. 1016/B978-0-12-408051-5.00005-X

Yang L et al (2012) The proteomics of lipid droplets: structure, dynamics, and functions of the organelle conserved from bacteria to humans. J Lipid Res 53:1245-1253. doi:10.1194/jlr.R024117

Yang Z-K et al (2013) Molecular and cellular mechanisms of neutral lipid accumulation in diatom following nitrogen deprivation. Biotechnol Biofuels 6:67

Yoon K, Han D, Li Y, Sommerfeld M, Hu Q (2012) Phospholipid:diacylglycerol acyltransferase is a multifunctional enzyme involved in membrane lipid turnover and degradation while synthesizing triacylglycerol in the unicellular green microalga Chlamydomonas reinhardtii. Plant Cell 24:3708-3724. doi:10. $1105 /$ tpc.112.100701

Zhang R, Patena W, Armbruster U, Gang SS, Blum SR, Jonikas MC (2014) High-throughput genotyping of green algal mutants reveals random distribution of mutagenic insertion sites and endonucleolytic cleavage of transforming DNA. Plant Cell. doi: $10.1105 /$ tpc. 114.124099

Zweytick D, Athenstaedt K, Daum G (2000) Intracellular lipid particles of eukaryotic cells. Biochim Biophys Acta-Rev Biomembr 1469:101-120. doi:10.1016/s0005-2736(00)00294-7 